\title{
In-medium properties of light vector mesons
}

\author{
C Djalali $^{1}$, R Nasseripour ${ }^{1}$, D P Weygand ${ }^{2}$ and \\ M H Wood ${ }^{3}\left(\right.$ CLAS Collaboration $\left.{ }^{4}\right)$ \\ ${ }^{1}$ University of South Carolina, Columbia, SC 29208, USA \\ 2 Thomas Jefferson Accelerator Facility, Newport News, VA 23606, USA \\ ${ }^{3}$ University of Massachusetts, Amherst, MA 01003, USA
}

Received 22 January 2007

Published 6 July 2007

Online at stacks.iop.org/JPhysG/34/S495

\begin{abstract}
The photoproduction of vector mesons on various nuclei has been studied using the CEBAF Large Acceptance Spectrometer (CLAS) at Jefferson Laboratory. All three vector mesons $\rho \omega$ and $\phi$ are observed via their decay to $\mathrm{e}^{+} \mathrm{e}^{-}$. The possible in-medium effects on the properties of the $\rho$ meson are of particular interest. The $\rho$ spectral function is extracted from the data on carbon, iron and titanium, and compared to the spectrum from liquid deuterium, which is relatively free of nuclear effects. We observe no effects on the mass of the $\rho$ meson, some widening in titanium and iron is observed consistent with the collisional broadening.
\end{abstract}

(Some figures in this article are in colour only in the electronic version)

\section{Introduction}

Quantum chromodynamics (QCD), the theory of the strong interaction, has been remarkably successful in describing high-energy and short-distance-scale experiments involving quarks and gluons. However, applying QCD to low-energy and large-distance-scale experiments has been a major challenge. Although the rapidly increasing strength of the interaction in this latter case makes it impossible to apply perturbative techniques, the symmetries of QCD (such as chiral symmetry) provide guiding principles to deal with strong interaction phenomena. Various QCD-inspired predictions are now available in the non-perturbative domain, which can be tested experimentally at current hadron and electromagnetic facilities.

Hadron masses, for example the proton at $\sim 1 \mathrm{GeV} / c^{2}$, are much larger than the summed masses of their constituent quarks, which are a few $\mathrm{MeV} / c^{2}$, indicating that much of the hadron mass is generated dynamically. Hadron masses are somewhat effected by the spontaneous breaking of chiral symmetry. One of these QCD-'inspired' predictions is that in hot (finite temperature) and/or dense (finite density) matter, a chiral phase transition takes place and the broken chiral symmetry is restored resulting in a modification of the properties of hadrons (vector mesons in particular) in nuclear matter from their free-space values. This prediction

4 A list of members of the CLAS Collaboration is given at the end of this issue. 
has generated much interest, and there is an urgent need for experimental data to confirm or refute these predictions in this as yet largely unexplored domain.

At high temperature or pressure, chiral symmetry may likely be restored. At normal nuclear densities, partial restoration of chiral symmetry may affect the properties of hadrons, in particular masses and widths [1-6].

The first evidence of a medium-effected $\rho$ meson mass came from the CERES [7] and HELIOS/3 [8] Collaborations at CERN in 1995. The CERES Collaboration reported on measurements of low-mass $\mathrm{e}^{+} \mathrm{e}^{-}$pairs from $\mathrm{p}-\mathrm{Au}$ and $\mathrm{Pb}-\mathrm{Au}$ collisions. Di-lepton spectroscopy allows measurement of the in-medium properties without distortion due to finalstate interactions (FSI). While their proton-induced data could satisfactorily be accounted for by summing various hadron decay contributions, an enhancement over the hadronic contributions was observed for the $\mathrm{Pb}-\mathrm{Au}$ data in the mass range between 300 and $700\left(\mathrm{MeV} / c^{2}\right)$. The same year, theorists were able to account for the observations by using a relativistic transport model assuming a decrease in the mass of the $\rho$ meson [9]. It has been suggested that traditional effects, such as an in-medium-modified pion dispersion relation, may be able to provide enough enhancement.

A better understanding of hadron properties in a hot and/or dense environment is therefore one of the most important endeavours of hadronic physics today. The change of properties of the vector mesons in the medium is a hot topic that is currently being studied at RHIC (BNL) and HADES (GSI) and will be studied at ALICE (CERN) by measuring low-mass di-lepton production. Although vector mesons preferentially decay into pions, the large finalstate interactions of the pions with the nuclear medium makes it almost impossible to derive any direct information about the meson properties in the medium. In a heavy-ion collision the final di-lepton yield is obtained by integrating over different densities and temperatures, and discriminating between different scenarios of the in-medium modifications for the vector mesons is difficult.

In their initial stages, relativistic heavy-ion reactions originate far from equilibrium and the temperature and density evolve over time. However, all theoretical predictions of inmedium properties of vector mesons in photon- or pion-induced reactions allow one to study the hadron properties in an environment that is much closer to equilibrium (normal nuclear density and zero temperature). The predicted in-medium effects for the vector mesons by the different models are so large that they should have observable consequences already at normal nuclear density. An experiment like g7 is ideal to measure the photoproduction of vector mesons off nuclei and will give complementary information about the in-medium properties of mesons.

\section{Theoretical predictions}

Due to chiral symmetry restoration, the mass and width of vector mesons, such as $\omega \rho$ and $\phi$ are predicted to change with increasing density. Brown and Rho [3] (BR), starting from an effective Lagrangian approach at low energy and zero density, suggest the same Lagrangian at high density, but with the masses and coupling constants that are modified according to the symmetry constraints of QCD. They proposed an in-medium scaling law that predicts a decrease in the mass of the vector meson by about $20 \%$ in normal nuclear matter.

Hatsuda and Lee [4] (HL), based on QCD sum rule calculations, obtained the spectral changes of the vector mesons in the nuclear medium. Their calculations result in a linear decrease of the masses as a function of density $\rho$ :

$$
\frac{m_{\mathrm{VM}}(\rho)}{m_{\mathrm{VM}}(\rho=0)}=1-\alpha \frac{\rho}{\rho_{0}}, \quad \alpha=0.16 \pm 0.06
$$


While QCD-based models predict a large downward shift of the vector-meson masses, more 'conventional' processes such as in-medium re-scattering predict no change in the vectormeson mass but a substantial increase in the width of the meson. These effects are density dependent, and, if present, should be observed at normal nuclear densities. Consequently, one should be able to observe the medium modifications of the properties of the hadrons in pion-, proton- or photon-induced reactions. Models based on nuclear many-body effects predict a broadening in the width of the $\rho$ meson with increasing density. This prediction is based on the assumption that many-body excitations may be present with the same quantum numbers and can be mixed with the hadronic states $[5,6]$.

\section{Existing data}

An observation of a medium-modified $\rho$ meson mass has also been claimed by the KEK-PS Collaboration in an experiment where $12 \mathrm{GeV}$ protons were sent on $\mathrm{C}$ and $\mathrm{Cu}$ targets and the $\mathrm{e}^{+} \mathrm{e}^{-}$pairs were detected [10]. However, the clearest evidence is obtained for the $\phi$ meson. By selecting low-momentum $\phi$ mesons, the $\phi$ meson peak in the invariant mass spectrum develops a shoulder due to the possibly modified $\phi$ 's decaying in the nucleus. Since few $\phi$ 's decay inside the nucleus, one needs sufficient statistics after the momentum cuts to observe the shoulder.

Very recently, the Crystal Barrel/TAPS collaboration has reported a downward shift in the mass of the $\omega$, where the analysis focused on the $\pi^{0} \gamma$ decay of low-momentum $\omega$ mesons photoproduced on a nuclear target [11]. The $\omega \rightarrow \pi^{0} \gamma$ channel is a very 'clean' channel for studying $\omega$ mesons since the branching ratio for $\rho \rightarrow \pi^{0} \gamma$ is two orders of magnitude smaller. Data were taken for two nuclear targets $\left(\mathrm{H}_{2}\right.$ and $\left.\mathrm{Nb}\right)$ and compared after subtracting a huge background. An enhancement was found towards lower masses for $\omega$ mesons produced on the $\mathrm{Nb}$ target. One concern about this experiment is the $\pi^{0}$ re-scattering in the nuclear medium which could distort the invariant mass spectrum.

Recently, the NA60 experiment at CERN SPS has studied low-mass muon pairs in 158 A GeV In-In collisions (see these proceedings). A strong excess of pairs is observed above the expectation from the neutral meson decays. The high statistics and good mass resolution of about $2 \%$ have allowed isolating the excess by subtraction of the known sources. The comparison of the $\rho$ spectral function with the prediction of the Rapp/Wambach for broadening and that of Brown/Rho for the shift in the mass of the $\rho$ shows no shift in mass but a broadening in the width of the $\rho$.

The latest medium modification results reported in relativistic heavy-ion experiments are not consistent with the KEK and TAPS conclusions. Experiments, such as g7 at JLab, that look for medium modifications in normal nuclear density at equilibrium are needed to disentangle the different mechanisms. All these experiments are yielding results complementary to each other and offer excitement, but no clear consensus has yet emerged between the various analyses and the question of the density-induced modifications on the properties of vector mesons is still wide open.

\section{The CLAS experiment g7}

The data for this study were taken in 2002 using the CEBAF accelerator and the CLAS detector located in the Hall-B of the Jefferson Laboratory. A comprehensive description of CEBAF, the Continuous Electron Beam Accelerator Facility, can be found in [12], while [13] gives an elaborate account on the CLAS, the CEBAF Large Acceptance Spectrometer, and other Hall-B 


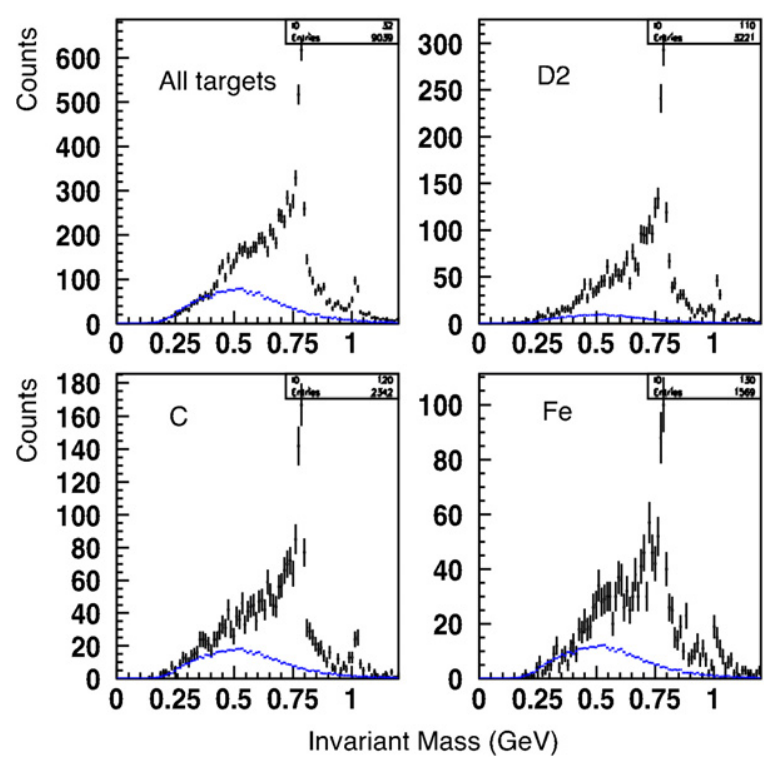

Figure 1. Normalized combinatorial background (blue) for all and individual targets compared to data (black).

equipments. CLAS is a nearly $4 \pi$-detector which was designed to track charged particles with momenta greater than $200 \mathrm{MeV} / c$ over the polar angle range from $8^{\circ}$ to $142^{\circ}$, while covering up to $80 \%$ of the azimuth. The detector is made of three regions of drift chambers, time-offlight scintillators, Cerenkov counters (CC) and electromagnetic calorimeters (EC). The $\mathrm{e}^{+} \mathrm{e}^{-}$ event selection and the rejection of the very large $\pi^{+} \pi^{-}$background were done through cuts on the EC and the CC.

The target contained a liquid deuterium (LD2) cell and seven solid foils, each with a $1.2 \mathrm{~cm}$ diameter. The separation between targets was $2.5 \mathrm{~cm}$; the target nucleus was determined by the position of the production vertex.

Lepton pair production has a background of random combinations of pairs due to the uncorrelated sources. The most salient feature of the uncorrelated sources is that they produce the same-charge lepton pairs as well as oppositely charged pairs. The same-charge pairs $\left(\mathrm{e}^{+} \mathrm{e}^{+}\right.$ and $\mathrm{e}^{-} \mathrm{e}^{-}$) provide a natural normalization of the uncorrelated background. This combinatorial method has successfully been used in the past for measurements involving opposite-sign pairs of pions or muons $[14,15]$.

The combinatorial background is statistically approximated by an event-mixing technique. The electrons of a given event are combined with positrons of another event, as the two samples of electrons and positrons are completely uncorrelated. This produces the phasespace distribution where electrons and positrons are actually from different processes but lying in the same event.

The mixed opposite-charged lepton chosen from samples of uncorrelated events was used to estimate the shape of the combinatorial background.

The result is shown in figure 1 for the individual targets as well as the total data.

\section{Results and discussion}

To simulate each physics process, a realistic model was employed and corrected for the CLAS acceptance. The events were generated using a code based on a semi-classical 


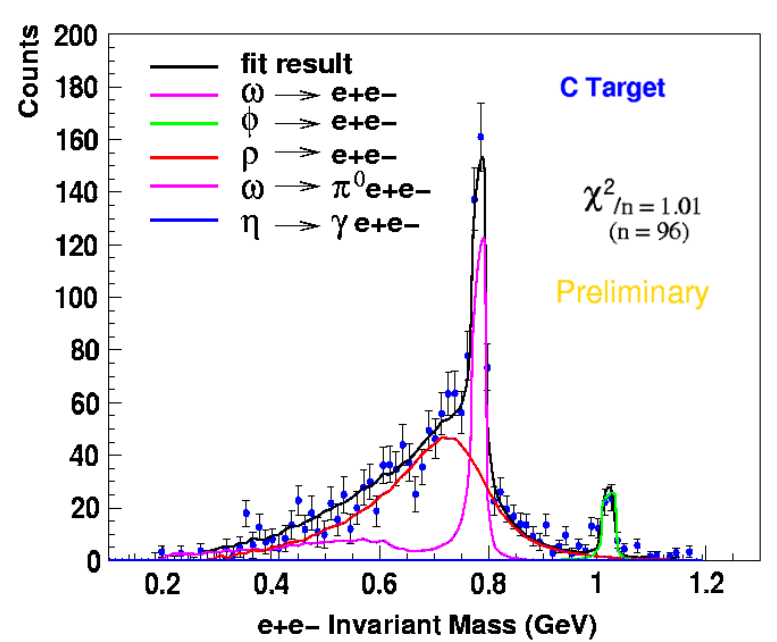

Figure 2. Results of the fit to the $\mathrm{e}^{+} \mathrm{e}^{-}$invariant mass obtained for $\mathrm{C}$ data. Curves are Monte Carlo calculations by the BUU model $[18,19]$ for various $\mathrm{e}^{+} \mathrm{e}^{-}$channels.

Table 1. Mass and width of the $\rho$ meson obtained from fits to the mass spectra (preliminary results).

\begin{tabular}{lllll}
\hline Target & $\begin{array}{l}\text { Mass }(\mathrm{MeV}) \\
\text { g7 data }\end{array}$ & $\begin{array}{l}\text { Width }(\mathrm{MeV}) \\
\text { g7 data }\end{array}$ & $\begin{array}{l}\text { Mass }(\mathrm{MeV}) \\
\text { BUU }\end{array}$ & $\begin{array}{l}\text { Width }(\mathrm{MeV}) \\
\text { BUU }\end{array}$ \\
\hline $\mathrm{D}_{2}$ & $770.3 \pm 3.2$ & $185.2 \pm 8.6$ & No BUU & No BUU \\
$\mathrm{C}$ & $768.5 \pm 3.7$ & $176.4 \pm 9.5$ & $773.8 \pm 0.9$ & $177.6 \pm 2.1$ \\
$\mathrm{Fe}$ & $779.0 \pm 5.7$ & $217.7 \pm 14.5$ & $773.8 \pm 5.3$ & $202.5 \pm 11.6$ \\
\hline
\end{tabular}

Boltzmann-Uehling-Uhlenbeck (BUU) transport model developed by the group of U Mosel at the University of Giessen which treats the photon-nucleus reactions as a two-step process [16]. In the first step, the incoming photons react with a single nucleon taking into account the effects of shadowing.

Then in the second step, the produced particles are propagated explicitly through the nucleus allowing for final-state interactions, governed by the semi-classical BUU transport equations. A rather complete treatment of the $\mathrm{e}^{+} \mathrm{e}^{-}$pair production from $\gamma \mathrm{A}$ reactions at Jefferson Lab energies has been carried out using this code [17].

The expected combinatorial background distributions are subtracted from the $\mathrm{e}^{+} \mathrm{e}^{-}$ effective mass distributions. The shape of the narrow $\omega$ and $\phi$ vector mesons and the $\omega$ Dalitz channel are well described by BUU model, and these distributions were fit to the data, then also subtracted, leaving just the experimental spectra of the $\rho$ mass. These fits for $\mathrm{C}$ and $\mathrm{Fe}-\mathrm{Ti}$ are shown, respectively, in figures 2 and 3.

The extracted $\rho$ mass distributions and the ratio to the deuterium data are then simultaneously fit with the suggested functional form of $1 / \mathrm{m}^{3}$ times a Breit-Wigner function [20-22].

The results of the fits are shown in figures 4-6 and tabulated in table 1 . The fits describe the data very well. The masses are consistent with the PDG values and the widths are consistent with the collisional broadening. We do not observe the doubling of the $\rho$ width reported by NA60 [23, 24]. Our results do not favour the prediction of Brown and Rho for the mass shift 


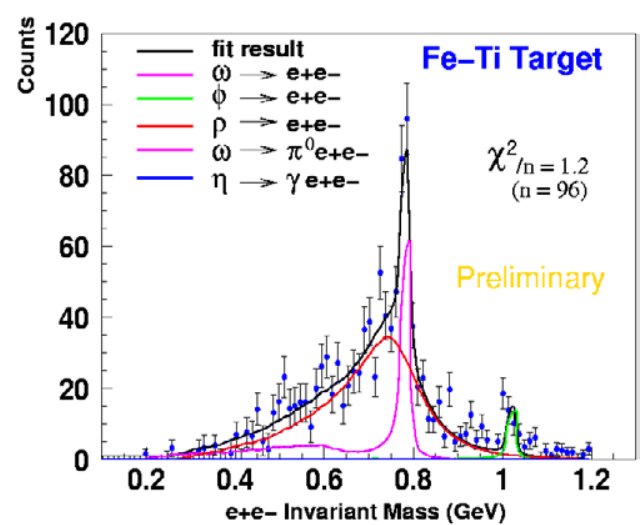

Figure 3. Results of the fit to the $\mathrm{e}^{+} \mathrm{e}^{-}$invariant mass obtained for Fe-Ti data. Curves are Monte Carlo calculations by the BUU model $[18,19]$ for various $\mathrm{e}^{+} \mathrm{e}^{-}$channels.

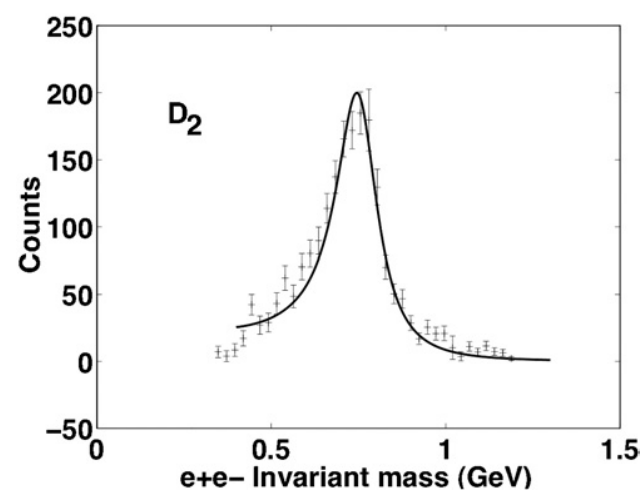

Figure 4. Extracted $\rho$ mass distributions and fit for deuterium.

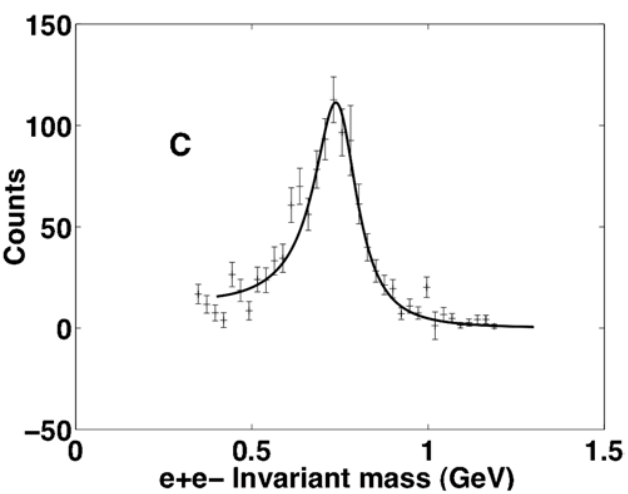

Figure 5. Extracted $\rho$ mass distributions and fit for carbon.

(20\%) or Hatsuda and Lee $(\alpha=0.16 \pm 0.06)$. Fit results on carbon data indicate no sensitivity to the mass shift predicted by the Hatsuda and Lee model, while Fe data do not favour the predicted shift. 


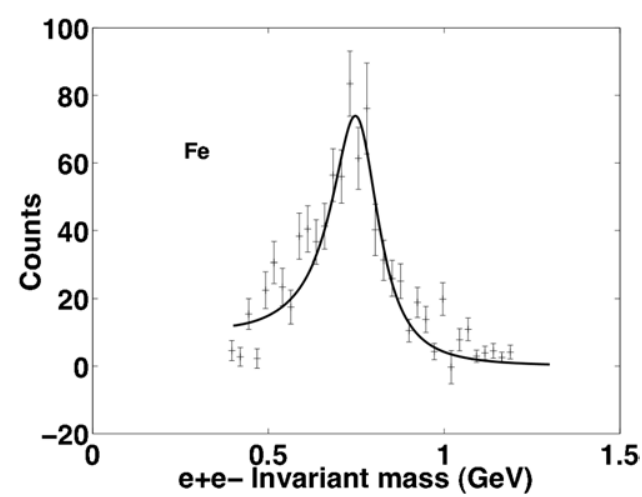

Figure 6. Extracted $\rho$ mass distributions and fit for iron-titanium.

\section{Acknowledgments}

The authors would like to thank U Mosel, P Muehlich, J Weil, O Buss and A Afanasev for providing us with theoretical support during this work. The US Department of Energy and the National Science Foundation supported this work. The Jefferson Science Associates, LLC, operates the Thomas Jefferson National Accelerator Facility for the United States Department of Energy under contract no DE-AC05-06OR23177.

\section{References}

[1] Bernard V and Meissner U G 1988 Nucl. Phys. A 489647

[2] Klimt S et al 1990 Phys. Lett. B 249386

[3] Brown G E et al 1991 Phys. Rev. Lett. 662720

[4] Hatsuda T and Lee S 1992 Phys. Rev. C 46 R34

[5] Herrman M et al 1992 Nucl. Phys. A $545267 \mathrm{c}$

[6] Rapp R et al 1997 Nucl. Phys. A 617472

[7] Agakichiev G et al 1995 Phys. Rev. Lett. 751272

[8] Massera M et al 1995 Nucl. Phys. A $59093 \mathrm{c}$

[9] Li G Q et al 1995 Phys. Rev. Lett. 754007

[10] Naruki M et al 2006 Phys. Rev. Lett. 96092301

[11] Trnka D et al 2005 Phys. Rev. Lett. 94192303

[12] Leemann C W, Douglas D R and Krafft G A 2001 Annu. Rev. Nucl. Part. Sci. 51413

[13] Mecking B A et al 2003 Nucl. Instrum. Methods A 503513

[14] Jancso G et al 1977 Nucl. Phys. B 1241

[15] Jouan B D et al 2002 IPNO-DR-02.015

[16] Muehlich P, Falter T, Greiner C, Lehr J, Post M and Mosel U 2002 Preprint nucl-th/0210079

[17] Effenberger M, Bratkovskaya E L and Mosel U 1999 Phys. Rev. C 60044614

[18] Effenberger M and Mosel U 2000 Phys. Rev. C 62014605

[19] Effenberger M, Bratkovskaya E L, Cassing W and Mosel U 1999 Phys. Rev. C 60027601

[20] Li G-Q et al 1996 Preprint nucl-th/9611037

[21] Effenberger M et al 1999 Preprint nucl-th/9903026

[22] O'Connell H B et al 1997 Prog. Part. Nucl. Phys. 39201

[23] Damjanovic S et al (for the NA60 Collaboration) 2005 Quark Matter

[24] Arnaldi R et al 2006 Phys. Rev. Lett. 96162302 